\title{
Coming back to the normal clinic: how has COVID-19 changed us?
}

\author{
Xiao Zhang ${ }^{1,2} \cdot$ Zhen Liu $^{1,2} \cdot$ Kuangyu Shi ${ }^{3,4} \cdot$ Xiaoli Lan $^{1,2}$ (D) \\ Received: 21 May 2020 / Accepted: 3 June 2020 / Published online: 12 June 2020 \\ (C) Springer-Verlag GmbH Germany, part of Springer Nature 2020
}

Dear Sir,

Until May 18th, more than 4 million cases worldwide were confirmed for the infection of COVID-19 leading to more than 310,000 deaths. As the initial epicenter, Wuhan had officially lifted the traffic control measures from April 8, which shed light on the gloomy situation. After 76 days of the lockdown, the city officially restarted. This is a milestone for the effective controls of COVID-19 in China. However, it is predicted that the recurrent outbreak may happen even after the elimination of the first pandemic wave [1].

Along with the reopening of the city, the number of patients undergoing nuclear medical examinations is gradually increasing. COVID-19 has resulted in a paradigm shift from traditional medical treatment towards online medical service. Patients can register and make an appointment online. They can communicate with the treating physicians including sending pictures and videos of their condition. Physicians could give patients advice and prescribe medicines remotely. Though information bias may exist and humanistic care partially lost, the online medical service can effectively reduce the exposure risk as well as save time. COVID-19 also brings changes to nuclear medicine department. Here, we share our current inspection

This article is part of the Topical Collection on Letter to the Editor

Xiaoli Lan

xiaoli_lan@hust.edu.cn; LXL730724@hotmail.com

1 Department of Nuclear Medicine, Union Hospital, Tongji Medical College, Huazhong University of Science and Technology, No. 1277 Jiefang Ave, Wuhan 430022, China

2 Hubei Key Laboratory of Molecular Imaging, Wuhan 430022, China

3 Department of Nuclear Medicine, University of Bern, Bern, Switzerland

4 Department of Informatics, Technical University of Munich, Munich, Germany procedures and hope to assist the nuclear medicine clinical work safely during this period.

Before performing radionuclide imaging, all patients and accompanies are demanded to test the antigen and antibody of SARS-CoV-2. If the patient needs to be hospitalized for radionuclide therapy, additional lung CT examination is required. The patients with positive results should be reported to the relevant infection or medical management departments, and further diagnosis or treatment will be arranged. Patients with negative results could proceed to examination reservation. Appointments are made online, including collecting clinical and epidemiological history. All patients are scheduled with different time sections.

In our department, we developed a non-contact nuclear medicine imaging appointment software. Through this software, patients can input general information such as name, gender, age, and so on; upload the test results of SARSCoV-2; input medical history; or upload the whole processes of diagnosis and treatment. After the patients provide all the information, the doctors can process the appointment in the background, arrange the imaging time for patients, and inform the patients of the examination time and considerations by SMS.

Before getting back to work, all medical staffs are also needed to test the antigen and antibody of SARS-CoV-2. All staffs are required to learn the information about COVID-19 and the prevention measurements via videoconference software. Psychological supports are also necessary to relieve the mental stress of staff. Free counseling is easily accessed, including hotlines and online consultation. The staff should keep a physically safe distance in the workplace but stay socially connected and good communication [2]. Because of the patient backlog in the early stage, we extend our working time and group into teams working in separate shifts. All the staff needs to wear a mask and emphasize hand hygiene. In order to 
shorten the unnecessary contact time with the patients, video can be used to observe the patients' condition during the whole imaging procedures.
The figure (Fig. 1) shows the measurements we are taking during the radionuclide imaging, except for the detailed infection prevention which we and others have mentioned before [3-5].
Fig. 1 Prevention measurements taken for the radionuclide imaging during the post-epidemic period of COVID-19
The patients and the accompanies are required to test antigen and antibody SARS-CoV-2 testing before radionuclide imaging.

(1) Measure body temperature for each patient and accompanies.

(2) Instruct the patients and accompanies to wear the masks.
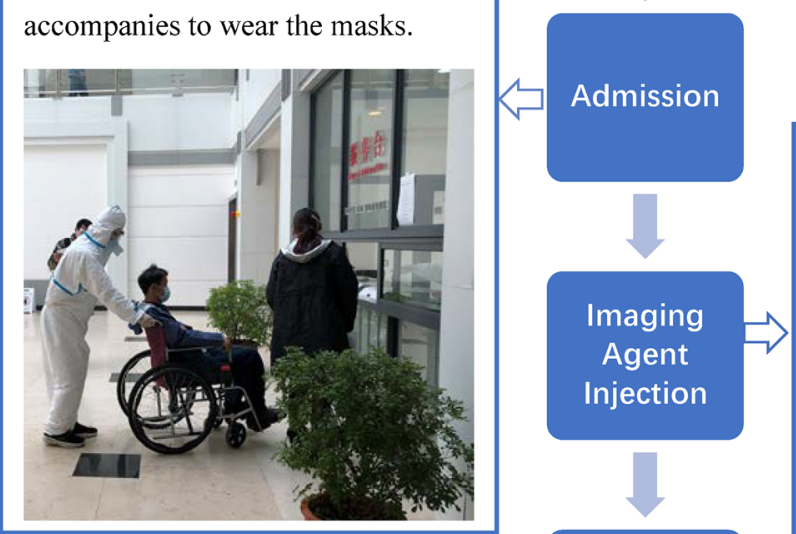

Pay attention to double protection: radiation and infection. Automatic injection robot would be better.

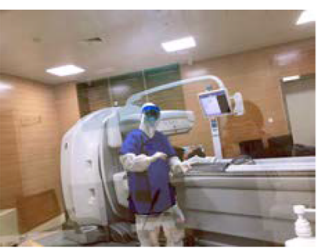

Waiting

After

(1) Patients need to keep a safe distance.

(2) Monitor the patients with video surveillance.

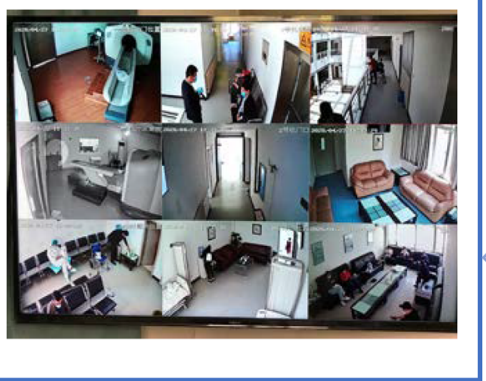

(1) Instruct the patients to wear a mask during the scanning.

(2) Observe patients during the scanning with video.

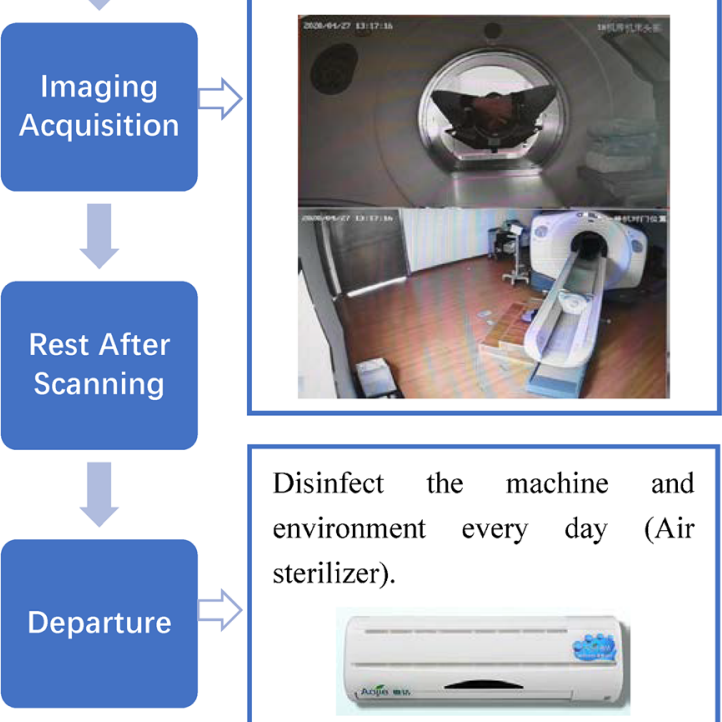




\section{Compliance with ethical standards}

Conflict of interest The authors declare that they have no conflict of interest.

Research involving human participants and/or animals None.

Informed consent None.

\section{References}

1. Leung K, Wu JT, Liu D, Leung GM. First-wave COVID-19 transmissibility and severity in China outside Hubei after control measures, and second-wave scenario planning: a modelling impact assessment. Lancet. 2020;395(10233):1382-93. https://doi.org/10. 1016/S0140-6736(20)30746-7.
2. Dickerson D. Seven tips to manage your mental health and wellbeing during the COVID-19 outbreak. Nature. 2020. https://doi. org/10.1038/d41586-020-00933-5.

3. Paez D, Gnanasegaran G, Fanti S, Bomanji J, Hacker M, Sathekge $\mathrm{M}$, et al. COVID-19 pandemic: guidance for nuclear medicine departments. Eur J Nucl Med Mol Imaging. 2020. https://doi.org/10. 1007/s00259-020-04825-8.

4. Huang HL, Gnanasegaran G, Paez D, Fanti S, Hacker M, Sathekge $\mathrm{M}$, et al. Nuclear medicine services after COVID-19: gearing up back to normality. Eur J Nucl Med Mol Imaging. 2020. https://doi.org/10. 1007/s00259-020-04848-1.

5. Zhang X, Shao F, Lan X. Suggestions for safety and protection control in Department of Nuclear Medicine during the outbreak of COVID-19. Eur J Nucl Med Mol Imaging. 2020. https://doi.org/10. 1007/s00259-020-04779-x.

Publisher's note Springer Nature remains neutral with regard to jurisdictional claims in published maps and institutional affiliations. 